\title{
Tony Blair, le New Labour et la Troisième Voie - ou comment conquérir le pouvoir en Grande- Bretagne et en Europe
}

Tony Blair, New Labour and the Third Way, or how to Get to the Top in Britain and Europe

\section{François-Charles Mougel}

\section{OpenEdition \\ Journals}

Édition électronique

URL : http://journals.openedition.org/rfcb/705

DOI : $10.4000 /$ rfcb.705

ISSN : 2429-4373

Éditeur

CRECIB - Centre de recherche et d'études en civilisation britannique

Édition imprimée

Date de publication : 1 février 2002

Pagination : 78-94

ISBN : 2-911580-13-3

ISSN : 0248-9015

\section{Référence électronique}

François-Charles Mougel, « Tony Blair, le New Labour et la Troisième Voie - ou comment conquérir le pouvoir en Grande-Bretagne et en Europe », Revue Française de Civilisation Britannique [En ligne], $\mathrm{XI}-3$ | 2002, mis en ligne le 21 mars 2016, consulté le 30 avril 2019. URL : http:// journals.openedition.org/rfcb/705; DOI : 10.4000/rfcb.705

Ce document a été généré automatiquement le 30 avril 2019.

\section{c)}

Revue française de civilisation britannique est mis à disposition selon les termes de la licence Creative Commons Attribution - Pas d'Utilisation Commerciale - Pas de Modification 4.0 International. 


\title{
Tony Blair, le New Labour et la Troisième Voie - ou comment conquérir le pouvoir en Grande- Bretagne et en Europe
}

\author{
Tony Blair, New Labour and the Third Way, or how to Get to the Top in Britain \\ and Europe
}

François-Charles Mougel

Seconde philosophie politique de l'histoire britannique à s'incarner dans le nom de son inspirateur, le blairisme est-il une idéologie, à l'instar du thatchérisme ? Encensée par les uns pour ses avancées conceptuelles et son efficacité tactique, critiquée par les autres pour ses ambiguïtés ou sa vacuité, la Troisième Voie, qui en constitue l'expression la plus manifeste, forme-t-elle une doctrine globale ou n'est-elle qu'un outil opératoire, voire un simple masque utilitaire ${ }^{1}$ ? Pour en décider il faut partir d'un triple contexte. D'abord, celui de la transformation de la société britannique en une vaste classe moyenne privant le parti travailliste « traditionnel » de ses repères sociologiques existentiels. Ensuite, celui de la chute de l'URSS en 1991 et de la remise en cause de tout modèle marxisant qui a contraint le Labour à repenser ses dogmes fondamentaux à l'aune de la mondialisation et de la modernité. Enfin, celui du poids du conservatisme qui a dominé le $\mathrm{XX}^{\mathrm{e}}$ siècle et, sous sa version thatchérienne, a non seulement condamné les travaillistes à une longue opposition mais aussi façonné de nouveaux modes de pensée et de pouvoir incontournables, même pour ses opposants. Face à ces contextes divers qui représentent autant de contraintes, Tony Blair, élu leader du parti travailliste en juillet 1994, a dû se présenter simultanément en héritier, en contestataire et en continuateur. C'est donc par rapport aux enjeux du pouvoir - sa conquête et sa préservation - que son apport théorique doit être analysé, dans la pensée comme dans l'action, en référence permanente à ce qui s'impose comme la nouvelle donne politique du passage du Royaume-Uni au troisième millénaire : l'enjeu européen. 


\section{La Troisième Voie comme instrument de conquête du pouvoir (1994-1997) : les héritages}

2 La Troisième Voie se situe au centre, entre la gauche et la droite. Mais quelle gauche et quelle droite ? Clairement, entre la gauche de l'old Labour et la New Right thatchérienne. Mais, tout en s'en démarquant, il en assume sélectivement certains héritages.

3 En tant que leader, Blair est d'abord l'héritier d'un siècle de travaillisme ${ }^{2}$. Version britannique du socialisme, ce dernier est porteur d'une double culture : une culture de l'idéologie et une culture du pouvoir. Sur le plan doctrinal, son fondement réside dans les statuts de 1918 dont la Clause IV forme le pivot central. Exigeant «que soit assurée aux travailleurs manuels et intellectuels la jouissance totale du produit de leur travail et la répartition la plus équitable possible de ce travail sur la base de la propriété collective des moyens de production, de distribution et d'échange et du meilleur système possible de gestion et de contrôle par le peuple des secteurs industriels et de service", elle postule, dans son principe, une transformation globale de la société britannique comme l'affirmera derechef, en 1945, le manifeste électoral travailliste qui réclame «l'établissement du Commonwealth socialiste de Grande-Bretagne ».

4 Mais l'affirmation corollaire de "l'inévitabilité du gradualisme» et le respect affiché du primat de la démocratie parlementaire par le «père » des statuts, Sidney Webb, va réduire la portée marxisante du texte tout en facilitant la légitimation du travaillisme comme référent idéologique conforme au Westminster Model dominant. Partant, l'idéologie travailliste pourra servir de base aux grandes réformes de structures opérées lors des passages du Labour au pouvoir, notamment entre 1945 et 1951 et, plus secondairement, entre 1964 et 1970. Mais, parce qu'elle est duale dans sa finalité comme dans son expression, cette idéologie va donner naissance à un constant affrontement entre une "gauche socialisante " et une "droite révisionniste ", entre lesquelles la direction du parti, plutôt réformiste jusqu'en 1979, a dû constamment arbitrer avant de succomber à la poussée maximaliste de la gauche, entre 1979 et 1983. D'où, après le désastre électoral et la démission de Michael Foot, un long travail de recentrage entrepris par les deux prédécesseurs de Blair, Neil Kinnock et John Smith.

Où en est alors, en 1994, l'idéologie travailliste ? Fortement attachée à la justice et au progrès social, elle entend maintenir, voire étendre, le Welfare State et les services publics, bases de l'égalité des chances et de la solidarité collective et principaux vestiges du consensus inter-partisan d'après-guerre. Encore fidèle au dirigisme économique d'inspiration keynésienne, elle a accepté en 1988 l'économie de marché et en 1990 a renoncé à toute "doctrine socialiste de l'économie ». Soucieuse de l'identité et de la sécurité nationales, elle a répudié l'unilatéralisme et s'est ralliée aux valeurs occidentales et à la construction européenne (1989-94). Dès 1989, au congrès de Brighton, Kinnock donne le ton : le Labour se veut «européen, modéré, pragmatique ». Mais sans pour autant abandonner ses dogmes fondamentaux ni l'idéal d'une transformation de la société sous la houlette de l'État. Et c'est bien parce qu'il a partagé d'emblée cette vision réformiste, sans porter le passif de l'action, que Tony Blair, à la tête d'une nouvelle génération de députés et de militants, pourra l'emporter facilement lors de l'élection du leader de juillet 1994. Déjà l'idéologie s'affirme comme son terrain de bataille et comme sa base de pouvoir. 
6 Parallèlement, le Labour, né en 1900 de l'alliance entre le mouvement syndical et les sociétés de pensée socialistes, est également porteur d'une culture de pouvoir. Pouvoir interne d'abord. Parti de masse, le parti travailliste s'est doté de structures fondées sur les principes de la démocratie organique et du vote par mandats collectifs qui confèrent aux syndicats et aux militants un poids considérable dans les institutions et les décisions du mouvement. C'est dire l'enjeu essentiel que représente le contrôle des trois instancesclefs du parti : le Congrès, le Comité Exécutif National (NEC) et la direction du groupe parlementaire (PLP). Après avoir à peu près réussi, jusqu'en 1979, à préserver le caractère plutôt réformiste de son action, le parti est passé, en 1980, sous le contrôle de la gauche et des syndicats, suscitant dans l'opinion comme dans son propre électorat une image négative où se mêlent les spectres du " pouvoir syndical ", de la démagogie maximaliste et de l'inaptitude à gouverner qui vont valoir au Labour quatre défaites électorales successives entre 1979 et 1992. D'où, à nouveau, un long travail, mené par Kinnock et Smith, pour démocratiser et moderniser le parti, grâce à l'instauration, entre 1987 et 1993, du système OMOV (one member, one vote) et à l'élimination corrélative de la «loony left» ainsi que par une politique active de recrutement en parallèle au recentrage idéologique.

7 Au plan externe, la culture de pouvoir du Labour découle de sa position de second grand parti national britannique qui en fait non seulement l'émanation quasi hégémonique de toute la gauche mais aussi, dans le cadre de la démocratie bipartisane, l'adversaire principal des conservateurs. Au début des années 90 , le Labour cherche à se démarquer du souvenir mitigé laissé par les gouvernements MacDonald, Wilson et Callaghan et par les leaderships contestés de Gaitskell et Foot pour mieux se rattacher à son référent mythique : le Cabinet Attlee des années 1945-51, principal fondateur de la démocratie sociale britannique. Pour Blair, la victoire future ne peut passer que par la domination des forces potentiellement contestataires de son parti - l'aile gauche et la majorité des états-majors syndicaux - et par la reconquête de l'électorat. Sa maîtrise, par le biais du discours, des bases de pouvoir du leader impose par conséquent l'invocation de la tradition pour mieux en assurer la rénovation et le contrôle.

C'est donc de cette double culture, complexe, souvent contradictoire, qu'hérite Tony Blair le 21 juillet 1994. Telle quelle, elle ne lui laisse qu'un seul choix : poursuivre dans la voie des réformes engagées pour recentrer la doctrine et restructurer le parti. La «révolution Blair » - selon l'expression de Mandelson et Liddle ${ }^{3}$ - allait démarrer.

9 Mais, pour parvenir à cette opération, base de la reconquête du pouvoir, Blair allait devoir tenir compte d'un second héritage, lui-même issu d'une autre " révolution »: celui $\mathrm{du}$ thatchérisme. Cet héritage est dual, et, partant, utilisable, aux yeux du leader, simultanément en négatif et en positif. Au négatif, le culte de l'individualisme et la négation du concept de "société ", la célébration de l'argent-roi et l'acceptation des inégalités, l'unionisme et le refus de la spécificité des "nations britanniques», l'europhobie, croissante sous John Major, l'abandon des services publics aux forces du marché, la politisation «droitière » de l'État et des institutions, le rejet du consensus et des pouvoirs intermédiaires. Au positif, l'attaque contre toutes les formes de " collectivisme », l'orthodoxie monétaire et budgétaire, le repli de l'État sur son domaine régalien, la législation du contrôle syndical, le nationalisme, la défense de l'Europe des États-nations, le lien avec les États-Unis, le maintien de «la loi et de l'ordre», la définition d'une nouvelle méritocratie et la lutte contre les corporatismes, l'acceptation du passage au post-industrialisme et à la mondialisation, le renforcement de l'autorité du 
premier ministre et de son réseau de pouvoir. Au positif encore, l'idée que, par l'incantation rhétorique, un parti peut élargir sa base sociologique et idéologique bien audelà de ses frontières traditionnelles: Mme Thatcher n'avait-elle pas su mobiliser de 30 à $40 \%$ du vote ouvrier et les deux tiers de celui des classes moyennes autant par son discours que par ses actes, donnant ainsi à son parti dix-huit années de pouvoir continu? Par conséquent, si le parti conservateur devait être battu, il fallait aussi tenir compte de ses acquis et de son influence dans les mentalités collectives. En condamnant ses erreurs, le Labour pouvait reconquérir le pouvoir, tout en utilisant certains de ses acquis. Il pouvait aussi légitimer sa rénovation. Tout serait affaire ici de personnalité et de communication.

\section{Le syncrétisme blairiste : la gestion des acquis}

10 La première étape de la "révolution Blair " a été celle de la création du New Labour. Il s'agit d'abord d'exorciser la référence au socialisme. Dès son maiden speech du 6 juillet 1983, Blair se défend de toute approche doctrinaire - sous-entendu marxiste ou "gauchiste »- et présente sa version du socialisme comme un système de valeurs consensuel :

I am a socialist not through reading a textbook that has caught my intellectual fancy, nor through unthinking tradition, but because I believe that, at its best, socialism corresponds most closely to an existence that is both rational and moral. It stands for cooperation, not confrontation; for fellowship, not fear. It stands for equality, not because it wants people to be the same but because only through equality in our economic circumstances can our individuality develop properly. British democracy rests ultimately on the shared perception by all the people that they participate in the benefits of the common weal ${ }^{4}$.

11 Cette vision d'un socialisme communautariste préservant l'égalité des chances et la liberté de l'individu se retrouve largement développée dans la synthèse programmatique publiée par Blair en 1996 New Britain. My vision of a young country. Il y défend alors l'idée du socialisme comme un "social-ism». Tel est l'essence du socialisme pour Blair : un retour aux racines du terme - c'est d'ailleurs la signification première qu'il convient de donner de son « radicalisme »- comme type d'organisation de l'homme en société et non comme doctrine téléologique de l'humanité.

Désormais, il peut approfondir l'aggiornamento doctrinal lancé par Kinnock à partir de 1983 et qui s'incarne dans le texte Democratic Socialist Aims and Values de 1988. S'y lisent le rejet de toute définition classiste, égalitariste ou statiste du projet travailliste et, au contraire, l'affirmation d'une approche unanimiste, ouverte et démocratique: "The true purpose of democratic socialism and therefore the true aim of the Labour Party, is the creation of a genuinely free society in which the fundamental objective of government is the protection and extension of individual liberty ${ }^{5}$. Comme le soulignera Blair, la coupure essentielle est faite vis-à-vis du old Labour (la minuscule est significative) et la route vers le progressisme est ouverte. Le choix est désormais clair : il s'agit de savoir " how to transcend labourism without betraying the labour interest $»^{6}$. Pour ce faire, Blair propose la formule du New Labour (la majuscule est aussi significative) qui assume l'héritage sans aliéner le futur. L'héritage invoqué est celui du cabinet Attlee de 1945-51 - «the record of this government makes me proud to call myself a democratic socialist " ${ }^{7}$. L'objectif d'avenir est celui d'un "national renewal », fondé sur les valeurs de communauté, d'entraide, de besoin partagé d'effort, de 
fierté collective et d'individualisme solidaire précise Tony Blair qui conclut : «that is what socialism means to me $»^{8}$.

13 C'est dans cette perspective que, sitôt élu leader, Blair va s'employer à accélérer la rénovation de son parti. Comme le souligneront Mandelson et Liddle: «New Labour is not just a different route for the left to take alone but a concept which joins the left to the centre of British politics and is all the stronger for that ${ }^{9}$ ". D'abord en obtenant la refonte de la Clause IV dans le sens du « socialisme éthique » qui est pour lui la source même du travaillisme. Ce sera chose faite en 1995. Mêlant morale, économie et politique, le nouveau texte intègre les trois valeurs cardinales du blairisme, à savoir la dimension spirituelle et éthique, le solidarisme actif et l'imbrication entre capitalisme et démocratie :

Le parti travailliste est un parti démocrate-socialiste qui croit que, par nos efforts communs, nous obtiendrons plus qu'individuellement afin de fournir à chacun les moyens de réaliser ses vraies possibilités et à tous une communauté dans laquelle le pouvoir, la richesse et les chances sont entre les mains de la majorité et non d'un petit nombre, où nos droits reflètent nos devoirs, et où nous vivons ensemble librement dans un esprit de solidarité, de tolérance et de respect, par le biais d'une économie dynamique servant l'intérêt général, dans laquelle l'initiative du marché et la rigueur de la compétitivité s'unissent aux forces du partenariat et de la coopération pour produire la richesse nécessaire à la nation et à chacun sa chance de réussir avec un secteur privé florissant et des services publics de qualité... une société juste... une démocratie ouverte... un environnement sain ${ }^{10}$.

14 Sur cette nouvelle base doctrinale, Tony Blair va pouvoir consolider la démocratie interne au parti, généralisant le système OMOV, réduisant les liens avec les syndicats et visant, comme il l'annoncera au congrès de Blackpool d'octobre 1994, à faire du Labour «le parti de tout le peuple britannique $\|^{11}$. On comprend mieux ainsi comment Blair, qui a réussi à s'emparer du parti par le biais de l'idéologie, a pu aussi s'imposer comme stratège de sa mutation électoraliste. Conscient que le syndicalisme maximaliste, le gauchisme irréaliste et l'ouvriérisme décalé constituent autant de handicaps pour le parti travailliste à l'heure du rejet du pouvoir syndical par une opinion encore traumatisée par le winter of discontent de 1978-9, d'un reflux global dans toutes les démocraties occidentales des utopies marxisantes, et du passage de la Grande-Bretagne à la société post-industrielle de service, il a compris que seul un recentrage tactique permettrait à son parti de revenir aux affaires en éliminant tout à la fois le vieux travaillisme et le thatchérisme doctrinaire et en conquérant l'espace central abandonné par les excès de la nouvelle droite. D'où l'équation New Labour $=$ New Britain $=$ centrisme radical. C'est ce qu'il annonce lors du discours du 11 avril 1996 devant la Chambre de commerce américano-britannique de New York: "The solutions of neither the old Left nor the New Right will do. We need a radical centre in modern politics... and today's Labour Party - New Labour - is a party of the centre as well as the centre-left ${ }^{12}$. " Après quinze ans d'opposition, le Labour était prêt à le suivre : $95 \%$ des militants allaient ratifier le projet de programme New Labour, New Life for Britain. D'autant plus que Blair présentait habilement son projet sous la caution de trois figures tutélaires du Labour: Clement Attlee, l'homme du consensus et de la réforme, Nye Bevan, le héraut de la gauche et l'apôtre du communautarisme et Anthony Crosland, l'intellectuel «révisionniste» pionnier de l'adaptation du travaillisme aux exigences du monde moderne $^{13}$.

Toutefois, la victoire au sein du parti ne suffisait pas. Il fallait aussi élargir la capacité d'attraction du nouveau travaillisme au-delà de ses bases. Pour cela, un double combat sera mené. D’abord, pour systématiquement éliminer les conservateurs du centre en les 
condamnant comme nouvelle droite brutale, inégalitaire, réactionnaire, incompétente et corrompue. Sur ce terrain les difficultés de John Major et sa radicalisation unioniste, europhobe et ultra-libérale - sans parler de l'effet délétère de l'usure du pouvoir et du sleaze - allaient le servir en libérant une large fraction des classes moyennes de l'influence tory. Mais, dans le même temps, par une habile "opération de triangulation", bien décrite par J. Crowley ${ }^{14}$, Blair allait s'emparer des thèmes porteurs du torysme à savoir la loi et l'ordre, l'orthodoxie financière et budgétaire, l'anti-syndicalisme, la lutte contre les corporatismes, le pragmatisme modernisateur. C'est tout ce qu'il promettra dans le manifeste électoral de 1997 Because Britain Deserves Better. Ici, c'est la rhétorique de la nouveauté qui sert de support à ce positionnement: il ne s'agit pas de faire du "thatchérisme à visage humain » mais d'utiliser certaines de ses valeurs telles la liberté, le choix, la responsabilité, l'adaptabilité pour faire face à un avenir en pleine mutation que souligne bien le thème de "young country". En empruntant aux Tories le slogan de One Nation, la défense des valeurs spiritualistes et morales, le respect de la famille et des institutions sans oublier le réformisme populiste, il capte l'héritage du Disraëli de Young England comme celui de la Tory Democracy de Lord Randolph Churchill. De plus, pour confirmer son positionnement comme rassembleur global, il promet aux partis nationalistes la dévolution pour le pays de Galles et l'Écosse et une approche nouvelle de la question nord-irlandaise. Enfin, il accomplit une habile récupération du libéralisme institutionnel et théorique. D'abord en promettant aux libéraux-démocrates des réformes constitutionnelles - mode de scrutin, régionalisation, ouverture européenne, défense des services publics - susceptibles de consolider une éventuelle coalition. Ensuite, en se réclamant du réformisme avancé des libéraux radicaux du début du XXe siècle et des grands novateurs des années 1930-50. D'où l'invocation, au panthéon blairiste, des trois noms de Lloyd George, Keynes et Beveridge ${ }^{15}$ et le jeu subtil sur le second sens du terme « radical» accolé en permanence à celui de centre. Il s'agit bien ici de récupérer la fonction tribunicienne traditionnellement dévolue au parti libéral, d'annexer au passage les anciens du travaillisme qui ont fait sécession en 1981 avant de se rallier au SLD et de gagner le soutien d'une partie de l'intelligentsia " libérale », proche d'un autre repère du blairisme naissant : le parti démocrate américain. Restait à utiliser ces outils et ces acquis pour en faire une véritable machine idéologique et stratégique.

\section{La synthèse blairiste : valeurs et stratégie}

La caractéristique et la force du blairisme résident dans son refus de toute doctrine ou de tout dogme a priori et dans sa volonté de réformer profondément le Royaume-Uni dans la perspective du futur. C'est ce que postule le manifeste électoral de 1997: "We will be a radical government. But the definition of radicalism will not be that of doctrine, whether left or right, but of achievement. New Labour is a party of ideas and ideals but not of outdated ideology. What counts is what works. The objectives are radical. The means will be modern ${ }^{16}$. " Récusant le passif de la "vieille gauche» tout en rejetant le surnom de "Tory Blair», le nouveau leader, appuyé sur ses «think tanks» (Institute for Public Policy Research, Demos) et ses "spin doctors» (D. Milliband, P. Mandelson, P. Gould, A. Campbell), entend subsumer et transcender les héritages et les acquis pour bâtir ce qui ne sera pas une nouvelle idéologie mais un système conceptuel susceptible de porter le New Labour au pouvoir sans contraindre son avenir. Ce sera la Troisième Voie. Cette dernière constitue donc le 
vecteur qui a permis, selon l'expression de E. Shaw, de faire passer le travaillisme du "party programmating" au " party positioning ${ }^{17}$.

La Troisième Voie est avant tout un code de valeurs qui puise dans l'héritage des penseurs libéraux du début du XX siècle (L. T. Hobhouse, T. H. Green, J. A. Hobson ou A. Marshall) comme dans les travaux plus récents de J.Gray, A.Giddens, J. Rawls, A. Etzioni ou $\mathrm{J}$. Macmurray ${ }^{18}$. Il en ressort quelques référents essentiels, à savoir l'égalité de tous, l'égalité des chances, la responsabilité, le communautarisme, la liberté, le choix, la justice, le progrès, l'éthique, la modernité, l'écologie et le messianisme. Le tout avec de fortes références à l'humanisme philosophique et à l'espérance chrétienne qui sous-tendent l'optimisme et le volontarisme si propres à la psychologie de Tony Blair. Cet objectif va s'incarner dans un projet unanimiste: celui de la "stakeholder society ${ }^{19}$ annoncé dès janvier 1996. Articulé sur le concept de One Nation, ce projet de société de participation repose sur l'idée de contrat global : contrat entre les citoyens eux-mêmes dont les droits déterminent les devoirs, contrat entre les citoyens et l'État qui doit être à la fois leur guide et leur serviteur, contrat entre la société et le marché puisque l'économie est à la base de la croissance, du plein emploi, du Welfare State et du progrès social, ce qui suppose un partenariat collectif entre entrepreneurs et employés, entre producteurs et consommateurs, entre bénéficiaires et contributeurs.

18 La stakeholder society est tournée vers l'avenir comme le postule le manifeste électoral de 1997: " Our aim is no less than to set British political life on a new course for the future ». Ce futur étant imprévisible, voire dangereux - c'est la théorie du risque chère à A. Giddens - cela évite au New Labour de trop se lier les mains. Ainsi, les quatre enjeux essentiels définis par Blair dans son ouvrage de $1996^{20}$ - améliorer les conditions de vie grâce à la croissance économique, construire un nouvel ordre social fondé sur la solidarité, décentraliser et rendre responsable le système politique, renforcer la position du Royaume-Uni dans le monde - peuvent être déclinés en un programme largement consensuel et adaptable, conforme au paradigme du centrisme radical. C'est exactement le sens du manifeste électoral de 1997 qui énumère ainsi, dans l'ordre, les priorités suivantes: «We will make education our number one priority... promote personal prosperity for all... help create successful and profitable businesses...get the unemployed from welfare to work... save the National Health Service... be tough on crime and the causes of crime... strengthen family life... help you get more out of life... clean up politics... give Britain leadership in Europe ».

19 Accompagné de la promesse de conserver les engagements budgétaires et fiscaux du gouvernement Major, ce programme permettait tout à la fois à Blair de gagner «la bataille des idées $»^{21}$ et de séduire la majorité de l'électorat. Le $1^{\text {er }}$ mai 1997, avec 43,2 \% des suffrages, il obtient 419 sièges sur un total de 659 soit la plus forte majorité jamais obtenue par le Labour, les Tories encaissant avec $30,7 \%$ des voix et 165 sièges une de leurs pires défaites. Mieux même, les travaillistes l'emportent dans toutes les régions, chez les hommes comme chez les femmes, dans toutes les générations sauf chez les + de 65 ans et dans toutes les classes sociales à l'exception des catégories supérieures même si, là aussi, ils progressent nettement, signe que les élites ont commencé de basculer vers le New Labour, à l'instar de la presse populaire d'ordinaire plutôt favorable aux conservateurs (voir la conversion spectaculaire du Sun entre 1992 et 1997).

20 Portée par un leader jeune, charismatique et innovateur, la rhétorique de la Troisième Voie a offert au New Labour une victoire trop longtemps attendue. Elle s'est donc révélée comme une indispensable machine à gagner le pouvoir. Généreuse dans les principes comme dans les propositions, elle peut aussi se lire comme le fruit d'une ambition 
personnelle dévorante et d'un machiavélisme retors. Si l'on s'en tient aux résultats, les militants du Labour et les électeurs n'y ont, semble-t-il, pas cru. Par conséquent peut se poser la question corollaire : la Troisième Voie allait-elle permettre aux travaillistes et en premier lieu à son inspirateur, Tony Blair, de le garder? Car de même qu'il s'est servi des recettes des Tories et des démocrates américains pour gagner la bataille, Blair a d'emblée pensé, comme eux, son programme sur le long terme : alors, sur quelles bases?

\section{La Troisième Voie comme vecteur de la conservation du pouvoir (1997-2001)}

21 À compter de la victoire de 1997, la Troisième Voie s'impose comme la référence majeure de la politique britannique, aussi bien au sein du parti travailliste que par rapport aux autres philosophies partisanes. Mais elle ne le fait pas comme idéologie ni comme contrainte. Elle le fait d'abord comme rhétorique de la nouveauté : "tout est nouveau» déclarera Tony Blair, ce qui permet de théoriser l'adaptabilité comme norme essentielle de la décision: «Nous devons être infiniment adaptables et faire preuve de la plus grande imagination. Il n'y a pas de préalables idéologiques. " Elle le fait ensuite comme dynamique : elle se construit en s'accomplissant, chaque réforme étant justifiée par l'action ellemême. Elle le fait enfin comme légitimation permanente, ce qui autorise le recadrage constant des politiques suivies par rapport aux engagements pris comme par rapport au projet d'avenir. Et c'est ici que la dimension « européenne » se surimpose à la dimension proprement «britannique " dans un processus récurrent d'autojustification et d'autopromotion. Le tout dans la perspective du Millennium qui constitue un atout psychologique supplémentaire et, surtout, dans la perspective du second mandat qui s'affiche, très vite, comme l'objectif essentiel du New Labour. C'est donc successivement comme centrisme, comme radicalisme et comme messianisme que l'on va retracer, en tant que construction intellectuelle, le bilan du premier gouvernement Blair ${ }^{22}$.

\section{La Troisième Voie comme centrisme}

Il s'agit ici de reprendre les acquis du thatchérisme tout en se démarquant de ses principes afin de mieux enfermer les Tories dans un bunker idéologique les condamnant à une opposition de longue durée. Le principal terrain d'action sera d'ordre économique et social. Le gouvernement Blair poursuit les grands chantiers de Thatcher et Major, rejetant les méthodes néo-keynésiennes, responsables des cycles stop/go - ou boom/bust antérieurs, responsables du retard britannique et adopte, sans les nommer, les méthodes monétaristes. Sur quatre ans le bilan est clair : la Banque d'Angleterre est devenue semiindépendante tout en contribuant à la solidité du sterling, l'orthodoxie budgétaire a été confortée au point de dégager des excédents, l'inflation a été contenue aux alentours de $2 / 2,5 \%$ par an, les privatisations ont été poursuivies (la Tote, le métro londonien, le contrôle aérien, les transports ferroviaires), la croissance a été encouragée et maintenue entre 2,5 et plus de $3 \%$ par an, le désengagement normatif et financier de l'État a été renforcé, l'appel au secteur privé en matière de services publics a été approfondi (Private Finance Initiative), le chômage a été réduit de 6 à 3,2 \%, le Welfare State a été réorganisé dans le sens de la rentabilité, la politique contre la délinquance a été accentuée (Crime and Disorder Act de 1998) et, enfin, la législation de contrôle syndical a été confirmée. 
S'agit-il pour Blair, comme on l'en a accusé, de faire simplement « du thatchérisme à visage humain »? Non point. Car la politique centriste ne s'affiche pas comme continuité mais comme réalisme novateur : «La gestion de l'économie n'est ni de gauche ni de droite. Elle est bonne ou mauvaise. Ce qui compte, c'est ce qui marche ", affirmera le premier ministre lors de son discours devant l'Assemblée nationale française le 24 mars 1998 tout en ajoutant, en guise de satisfecit: «elle est bien gérée par la gauche aujourd'hui »" ${ }^{23}$. En outre l'appel systématique à la "culture d'entreprise ", dès l'école et dans tous les aspects de la vie collective, est présenté comme un atout, permettant aux citoyens de se réapproprier, comme le souligne J. Crowley ${ }^{24}$, tout à la fois l'État et le marché selon la formule, empruntée à L. Jospin : "oui à l'économie de marché, non à la société de marché "25. Mieux même, chaque réforme est soigneusement replacée dans le cadre d'un projet global d'avenir, qu'il s'agisse de la réforme du marché du travail au nom de la flexibilité et de l'employabilité (programme Welfare to Work) ou de la politique fiscale - la baisse des impôts directs étant toutefois plus que compensée par l'augmentation des indirects ce qui aboutit à une légère hausse des prélèvements obligatoires de 35,5 à $37 \%$ du PNB - ou de la lente adaptation des services publics. Le texte de présentation du Livre vert sur la réforme du Welfare State est tout à fait caractéristique en ce sens :

La réforme nous redonne une véritable cause nationale, elle nous ouvre une perspective plus large qui fera de notre pays le modèle de la nation développée $d u$ $\mathrm{XXI}^{\mathrm{e}}$ siècle avec une gestion saine et stable de l'économie, le dynamisme et l'esprit d'entreprise, un niveau d'entreprise, un niveau d'éducation et de créativité sans égal dans le monde et un État-Providence fait pour encourager les projets et la réussite ${ }^{26}$.

Cette stratégie permet de justifier le lien entre le monde des affaires et le gouvernement "le New Labour est le parti du business ${ }^{27}$ »-, de rassurer Middle England en écartant tout risque de retour aux dérives de l'old Labour en matière fiscale et syndicale tout en préservant son rôle central - «le Labour est le parti des classes moyennes » comme l'affirmera J. Prescott - de ne pas mécontenter les nouveaux électeurs du New Labour puisque les actes sont conformes au manifeste électoral et, surtout, de priver les Tories de tout instrument de critique sérieux puisque l'essentiel des acquis du thatchérisme est maintenu. En outre, cette politique s'articule avec la rhétorique de One Nation annoncée dès le discours du trône du 14 mai 1997 : "Mon gouvernement a l'intention de gouverner dans l'intérêt de la nation tout entière. Nous sommes le parti d'une seule nation. C'est un non-sens de dire qu'il n'existe pas de différence entre les partis ... [mais le changement] sera conduit sans considération de dogme et de doctrine. $»^{28}$ Manière de s'emparer du thème conservateur de l'unité nationale tout en condamnant le sectarisme des Tories et le rejet par Mme Thatcher du concept même de société. Ainsi, ce positionnement centriste permet de transcender tous les clivages sociaux et idéologiques en se plaçant sous la bannière modernisatrice de l'intérêt général et la perspective du futur. C'est ce que soulignera A. Giddens dans son ouvrage sur la Troisième Voie (1998) :

L'objectif politique de la Troisième Voie est d'aider les citoyens à traverser les principales révolutions de notre temps, la mondialisation, les transformations de nos vies personnelles et de nos rapports avec la nature. La politique de la Troisième Voie doit adopter une attitude positive envers la mondialisation mais... uniquement comme un phénomène qui dépasse largement le marché global ${ }^{29}$. 


\section{La Troisième Voie comme radicalisme}

Parallèlement au centrisme, Tony Blair a développé l'aspect radical de sa Troisième Voie. Son but est d'incarner l'esprit du changement que veut porter le blairisme tout en restant fidèle à l'esprit de la continuité, l'ambiguïté potentielle étant transcendée par la nouveauté, comme le soulignera le premier ministre en appelant de ses vœux, lors du congrès de Brighton, le 30 septembre $1997:$ «La renaissance d'une nation et la nouvelle confiance en elle d'une Grande-Bretagne fondée sur les bonnes vieilles valeurs britanniques. Nous ne pourrons jamais être les plus grands. Nous ne serons sans doute jamais plus les plus puissants. Mais nous pouvons être les meilleurs... rien de moins que la nation-modèle du XXI ${ }^{e}$ siècle, un phare pour le monde $e^{30}$ ». Cette "révolution tranquille» que, selon ses propres termes ${ }^{31}$, le premier ministre met en place, est avant tout politique et sociale. Sur le plan politique, elle est principalement d'ordre constitutionnel. Elle passe par une réflexion sur le rôle futur de la monarchie, la réforme de la Chambre des Lords, la dévolution en Écosse et au pays de Galles, le rétablissement d'une autorité du Grand Londres avec un maire élu, une possible régionalisation de l'Angleterre, la résolution de la question d'Irlande, l'introduction du vote proportionnel pour le scrutin européen et les nouvelles assemblées écossaises et galloises et sa possible instillation dans le mode de suffrage législatif national, l'adoption d'une Charte des citoyens et, à terme, de la Charte européenne. Sur le plan social, elle s'exprime au travers du New Deal - la reprise de l'expression rooseveltienne n'est pas sans signification - qui vise au plan social et syndical à établir les droits et les devoirs des employeurs et des salariés, compensant l'employabilité imposée aux seconds par des concessions demandées aux premiers (salaire minimum, adoption de la charte sociale européenne, programme Fairness at Work en faveur des syndicats). S'y ajoutent des mesures concernant le logement, l'exclusion, la culture, et surtout l'éducation présentée comme la priorité $\mathrm{n}^{\circ} 1$ du gouvernement et le vecteur incontournable de la modernisation du pays.

Ces réformes permettent au New Labour de se démarquer des positions des conservateurs en offrant aux Britanniques un nouveau pacte d'identité nationale et en prônant l' "inclusive society». Elles peuvent séduire les libéraux-démocrates attachés à la décentralisation, au scrutin proportionnel et à la dimension européenne. Enfin et surtout, elles visent à satisfaire la majorité des travaillistes en favorisant l'émergence de nouvelles élites fondées sur la méritocratie, en satisfaisant des projets constitutionnels depuis longtemps sur les agendas du parti et en donnant une perspective dynamique au programme gouvernemental. Pour tenir tous ces objectifs, Tony Blair a dû imposer un style de pouvoir tout à la fois autoritaire et charismatique. Jamais la centralisation de la décision et du discours ni la présidentialisation des fonctions de premier ministre et de leader n'ont été aussi grandes au sein du gouvernement comme du parti. Pourquoi ? Parce qu'il ne faut qu'un discours unique qui surmonte les contradictions potentielles et les controverses possibles au nom de ce que Peter Mandelson nomme « la révolution en marche ${ }^{32}$ ", dont les buts principaux sont incessamment martelés: concilier croissance économique et justice sociale, renforcer le rôle de guide et de coordinateur de l'État, rénover le Welfare State, rétablir la dimension européenne de la politique du RoyaumeUni.

L'autoritarisme de Downing Street apparait ici comme une exigence de la Troisième Voie. En effet, cette dernière n'est pas sans limites ni sans contraintes. Limites dues à ses 
ambivalences et à sa modération fondamentale, contraintes liées à l'irruption de contingences imprévisibles ou aux conséquences contradictoires du télescopage du centrisme et du radicalisme. Ainsi, au sein du Cabinet la collégialité a été court-circuitée par le renforcement des réseaux de pouvoir du premier ministre, le contrôle politique sur le Civil Service s'est accentué et la médiatisation a imposé un "gouvernement par l'image » au seul bénéfice du premier ministre. Plus largement, le chef du gouvernement a entrepris de contrer les oppositions latentes ou renaissantes dans la gauche partisane et syndicale par une emprise croissante sur le NEC et le congrès comme sur les instances militantes (ce qui s'est traduit par une baisse du nombre des adhérents directs passé de 265000 en 1993 à 425000 en 1997 et retombé à 380000 en 2000) et s'est efforcé de réduire le rôle du TUC, non seulement comme force socio-économique mais aussi comme force politique. C'est pourquoi, au mépris de ses engagements en faveur de la démocratie interne et de l'esprit même de la décentralisation, Blair a cherché à imposer sa loi en Écosse, au pays de Galles et surtout à Londres où, à son grand dam, l'élection du leader de gauche Ken Livingstone en mai 2000 a pu apparaître comme un revers sérieux pour le premier ministre, sans parler des reculs électoraux sensibles aux divers scrutins locaux comme aux élections européennes de 1999. Dans un domaine où la gestion de l'événementiel est cardinale, l'impondérable constitue un risque majeur comme l'ont montré, outre la persistance du drame nord-irlandais, les catastrophes successives de la vache folle et de la fièvre aphteuse qui ont révélé plusieurs dysfonctionnements de la machine étatique.

Tony Blair a dû aussi affronter les conséquences sociales difficiles de sa politique libérale en matière économique avec la paupérisation accrue d'un quart de la population et la précarisation de nombreux secteurs et de maintes régions. Il a, enfin, dû faire face à la dégradation spectaculaire des services publics notamment dans les domaines de la santé, de l'éducation et des transports ferroviaires. Pour surmonter ces contradictions, voire ces échecs, que ponctuent parallèlement plusieurs crises ministérielles, Blair a réutilisé la rhétorique de la modernité et de la "révolution en marche ", autrement dit, rejeté sur les Tories l'héritage de la mauvaise gestion de l'État et des services publics en promettant de nouveaux investissements et de nouvelles réformes et en demandant à l'électorat par la défense systématique de son bilan, l'obtention d'un second mandat, plus « radical ». C'est tout le sens qu'il faut donner à la campagne électorale de 2001 et au programme travailliste qui la conditionne : Ambitions for Britain.

L'enjeu politique est ici vital pour le premier ministre dans la perspective de ce qui a toujours été son objectif : conduire son parti à une seconde, voire une troisième victoire électorale ouvrant, selon ses propres mots, un siècle de radicalisme, comme le $\mathrm{XX}^{\mathrm{e}}$ siècle avait été un siècle de conservatisme ${ }^{33}$. D'où, d'ailleurs, dans le discours, des modifications sensibles : l'expression de stakeholder society disparaît au profit de celle d'inclusive society, l'idéal d'égalité devient celui de méritocratie, la Troisième Voie s'affiche comme «politique progressive» et le radicalisme, tout en étant postulé comme "ancré au centre ", se pare d'attributs "révolutionnaires ». Tel est, d'ailleurs, le thème du discours de Blair au congrès de Bournemouth du 28 septembre 1999 :

Patience, prudence et modération sont les maîtres-mots de la Troisième Voie. On nous a accusés de mener la même politique que l'ancienne équipe conservatrice, c'est ridicule! Notre ambition est au contraire de libérer le pays et tous ses habitants des forces du conservatisme. Créer une nouvelle Grande-Bretagne de véritable égalité des chances n'est pas plus une trahison de l'histoire britannique que le nouveau travaillisme ne trahit les valeurs travaillistes. La Troisième Voie 
n'est pas un nouveau chemin entre conservatisme et progressisme, c'est une politique progressiste qui se distingue de tous les conservatismes, de droite comme de gauche ${ }^{34}$. maîtrisée du temps politique qui constituent le fondement du New Labour. C'est ici que le messianisme s'avère comme le complément indispensable de la stratégie de pouvoir de Tony Blair.

\section{La Troisième Voie comme messianisme}

Le messianisme est consubstantiel au blairisme. D'abord parce qu'il justifie l'entrée de Tony Blair en politique, au sein du parti travailliste :

Je suis né après la guerre... Seuls les pervers ne voyaient pas que l'expérience de l'Europe de l'Est était un désastre politique et économique. C'est parce que je croyais en une justice qui ne soit pas seulement individuelle mais sociale que je suis entré en politique. La vieille gauche croyait en un État-Providence non réformé ; la nouvelle droite veut le démanteler... la vieille gauche était en faveur du contrôle de l'État sur l'industrie, d'impôts et de dépenses élevés ; la nouvelle droite est pour le laisser-faire et le retrait de l'État de pratiquement tout. Le rôle que le Labour nouveau entend lui donner est différent, c'est d'équiper les gens et le business pour le changement par le partenariat ${ }^{35}$.

Ensuite, le messianisme permet de conduire le changement promis par-delà critiques, erreurs ou difficultés d'où l'autopromotion et l'autojustification récurrentes des actions entreprises. Le messianisme confine ici à la prédication: la presse n'ironise-t-elle pas, d'ailleurs, sur «le pasteur Blair»? Enfin, le messianisme, étendu à la dimension européenne, légitime, tout en l'amplifiant la révolution blairiste comme le premier ministre l'annonça dès 1997 : «C'est notre destin d'être à nouveau un leader en Europe : l'Europe a besoin de nous, car nous avons une vision, celle de l'Europe du peuple fondée sur le libre-échange, l'emploi et la justice sociale et la démocratie face à celle de l'Europe des bureaucrates ${ }^{36}$.

La dimension européenne de la Troisième Voie part d'un double constat. D'une part que la Grande-Bretagne ne saurait jouer seule face aux États-Unis, en dépit de sa "relation spéciale » avec Washington et des liens idéologiques et amicaux forts existant entre les néo-démocrates de Clinton et les travaillistes de Blair et qui semblent vouloir se prolonger avec la nouvelle administration républicaine de G.W.Bush. La tentative avortée d'une nouvelle Internationale de centre-gauche, lancée par le premier ministre britannique en février $1998^{37}$, échoua rapidement et le sommet de New York rassemblant Clinton, Blair, le Bulgare Stoïanov et l'Italien Prodi ne déboucha sur aucune structure entièrement vouée à la Troisième Voie ${ }^{38}$. Blair en tira pragmatiquement la leçon en renforçant l'ancrage européen de sa stratégie: «Il n'y a pas pour nous de choix entre l'Europe et l'Amérique. La Grande-Bretagne est importante aujourd'hui auprès de l'Amérique parce qu'elle est importante en Europe ${ }^{39}$ ».

L'Europe, pour Blair, constitue d'autre part un champ de pouvoir important, le moyen de grandir sa stature « interne » par une stature équivalente sur le continent. D'où, outre ses ouvertures vers la Russie de B. Eltsine et V. Poutine comme vers les ex-pays de l'Est qu'il importe de séduire comme candidats à l'OTAN et à l'UE, son offensive vis-à-vis des partenaires européens du Royaume-Uni. Sur ce point, l'évolution du discours blairiste forme un indicateur révélateur des transformations de la Troisième Voie comme concept philosophico-idéologique. Avec la France, T. Blair joue à la fois le partenariat classique de 
la coopération inter-étatique (voir l'accord de St. Malo de 1998) et la séduction idéologique contraignant J. Chirac et surtout L. Jospin à se positionner face à une Troisième Voie que le président accepte dans un sens «néo-libéral » alors que le premier ministre, soucieux de son ancrage à gauche, préférerait n'y voir que la forme nouvelle de la social-démocratie européenne ${ }^{40}$. Même stratégie vis-à-vis de l'Allemagne, que l'élection du leader du SPD, G. Schröder, en 1998 à la chancellerie a facilitée au point que, le 8 juin 1999, les chefs de gouvernement britannique et allemand ont publié un appel commun en faveur d'une réforme libérale de l'Union, le Neue Mitte germanique étant présenté comme l'équivalent du centrisme radical anglais ${ }^{41}$. Autre accord, encore plus révélateur, celui passé en juin et en octobre 2000 avec le conservateur espagnol Aznar ${ }^{42}$ et peut-être, demain, celui qui s'établira avec l'Italie de S. Berlusconi.

Cette stratégie tous azimuts, menée également avec les autres États européens, notamment l'Irlande, les pays nordiques ou le Portugal, se double d'un entrisme et d'un lobbying très sensibles dans toutes les instances européennes pour affaiblir l'axe francoallemand et faire prévaloir les thèses britanniques. Cette volonté de domination intellectuelle $\mathrm{du}$ futur européen se veut globale: affirmée dès le sommet de l'Internationale Socialiste de Malmö en juin 1997, elle a été confirmée lors de la rencontre des réformateurs de Florence de novembre 1999 qui rassemblait les progressistes européens et le Président Clinton autour du concept de Troisième Voie comme outil de conciliation entre mondialisation, croissance économique et justice sociale ${ }^{43}$. Elle a été réaffirmée en février 2001 par le ministre anglais des Affaires étrangères et futur président du Parti socialiste européen, Robin Cook, qui a assigné à ses partenaires, majoritaires dans l'UE, un sextuple défi qui résume, en fait, la vision blairiste de l'avenir $\mathrm{du}$ continent: "Traduire les valeurs internationalistes en partenariats internationaux, développer l'économie par l'essor des connaissances, faire de la justice sociale la condition du progrès économique, constituer un État actif, garantir une société ouverte nécessaire corollaire de la mondialisation, renforcer et réformer l'Union européenne ${ }^{44} »$. On ne saurait mieux postuler l'équation Troisième Voie = Nouvelle Europe.

En multipliant ainsi partenariats et convergence, Tony Blair a réussi à imposer à ses partenaires ses vues sur l'élargissement, l'approfondissement et la rénovation de l'UE dans un sens «national », « libéral » et pragmatique. Tout ceci, bien sûr, en conformité avec ses propres intérêts politiques et nationaux ${ }^{45}$. On comprend mieux ainsi sa stratégie sur l'euro. Après avoir confirmé son accord de principe en février $1999^{46}$, il refuse de se lier les mains, pour des raisons internes, sur un quelconque calendrier, préférant attendre l'issue du scrutin de 2001 et un futur référendum, pour fixer la date et les conditions de la fusion du sterling dans la monnaie unique. Dans toute cette gestion du dossier européen on voit bien s'esquisser la plasticité du paradigme de la Troisième Voie. Elle ne s'oppose pas à l'approche thatchérienne du discours de Bruges de 1988 tout en se montrant plus ouverte. Elle se situe entre la nouvelle droite et la social-démocratie sans concession aux tenants de l'interventionnisme étatique, elle se prononce pour un rassemblement d'États-nations sans appel au fédéralisme. Comme telle, elle légitime la position personnelle de Blair comme principal référent européen, ce qui ne peut, par ricochet, que légitimer sa position de dominateur idéologique en Grande-Bretagne même. Après tout, la rose rouge, symbole de la social-démocratie européenne, n'est-elle pas aussi celui de l'Angleterre? 


\section{En guise de conclusion}

37 166 aux conservateurs, est avant tout celle de Tony Blair et de sa stratégie. L'habile
gestion, dans un contexte pourtant délicat, de la campagne électorale, en stigmatisant les erreurs et les blocages des Tories tout en prêchant l'humilité sur un bilan travailliste que l'on ne cesse de valoriser, a réussi à minimiser - sauf au prix d'une forte abstention, de l'ordre de $40 \%$ - les limites, les faiblesses et les ambiguïtés de la Troisième Voie (n'est-ce pas l'Economist qui à la veille des élections de 2001 recommandait aux Britanniques de voter pour Blair, le seul vrai «conservateur » ? ${ }^{47}$ ). Mais c'était là, aussi, le coût estimé d'une analyse du pouvoir qui s'est révélée efficace. Après avoir permis à Blair de s'imposer comme leader du parti travailliste puis de conduire ce dernier à la victoire en 1997, elle lui a permis de garder le pouvoir dans sa formation comme dans le pays et de remporter un succès massif en 2001. Le concept reste encore opératoire puisque l'objectif sera, maintenant, de remporter une troisième victoire en 2006.

Le choix est désormais entre une gestion néo-libérale conforme à sa composante centriste ou une ouverture plus réformiste conforme à sa composante radicale ${ }^{48}$. Les promesses en faveur de la rénovation des services publics, du renforcement de la dimension européenne et de la lutte contre les barrières sociales dans le cadre de quatre nouvelles valeurs - service, responsabilité, devoir envers les autres et méritocratie - montre que la seconde approche pourrait être celle que suivra Tony Blair : « Now is the chance to build the future properly, to make the second term the basis for a radical programme of British renewal ", annonce-t-il en tête du manifeste électoral, ajoutant: "The choice Britain faces today is starker than in 1997. The Conservatives have swung further to the right. And in government, Labour's agenda has become increasingly bold and ambitious ${ }^{49}$ ». Ayant soigneusement étudié la politique de Margaret Thatcher, il ne lui a pas échappé que le second mandat de la dame de fer avait été le plus « révolutionnaire » : sans doute cet exemple sera-t-il médité, à condition de ne pas tomber dans les extrêmes qui ont conduit l'ancien premier ministre conservateur aux échecs du troisième mandat. Mais, en énumérant dix objectifs de réforme pour 2010, le premier ministre actuel ne veut envisager que la réussite et sur le très long terme. Si le succès de 2001 permet à Tony Blair de dépasser les limites présentes - mais assumées - de son projet et de transcender l'ambivalence volontaire qui est au cœur de sa "révolution tranquille ", preuve aura été faite que la Troisième Voie, en s'avérant simultanément comme une stratégie, une tactique et un messianisme, est bien l'instrument à la fois utopique et machiavélien de ce qui semble être son but: devenir l'inventeur d'un nouveau système de pouvoir pour le Royaume-Uni et l'Europe du XXI siècle. Assurément - et c'est ce qui peut déconcerter analystes ${ }^{50}$ et citoyens - la Troisième Voie apparait souvent comme un chemin sinueux, voire masqué, mais sa trajectoire est bien rectiligne. 


\section{NOTES}

1. Voir, entre autres: Pouvoirs, $n^{\circ} 93$, «Le Royaume-Uni de Tony Blair», Paris: Seuil, 2000 ; T. WHITTON (dir.), Le New Labour : rupture ou continuité ?, Rennes : P.U. de Rennes, 2000 ; E. AVRIL \& R.DAVIS (dir.), Comprendre la Grande-Bretagne de Tony Blair. Bilan d'une alternance politique, Villeneuve d'Ascq : P.U. du Septentrion, 2001 ; Témoins, n² 24, “New Labour...Old England ?", maijuin 2001.

2. M. CHARLOT, Le Parti travailliste britannique, Paris: Montchrestien, 1992; H. M. DRUCKER, Doctrine and Ethos in the Labour Party, Londres: G. Allen \& Unwin, 1979; C. COOK \& I. TAYLOR (eds.), The Labour Party, Londres : Longman, 1980; D. E. MARTIN \& D. RUBINSTEIN (eds.), Ideology in the Labour Movement, Londres: Croom Helm, 1979; H. PELLING, A Short History of the Labour Party, Londres: Macmillan, 1965; E. SHAW, The Labour Party since 1979. Crisis and Transformation, Londres : Routledge, 1994 ; M. PERRYMAN (ed.), The Blair Agenda, Londres: Macmillan, 1997. Voir aussi, comme sources : Let's Face the Future, Labour Manifesto 1945 ; Meet the Challenge, Make the Change, Labour Manifesto 1992.

3. P. MANDELSON \& R. LIDDLE, The Blair Revolution. Can New Labour Deliver ?, Londres: Faber \& Faber, 1996 ; S. DRIVER \& L. MARTELL, New Labour. Politics after Thatcherism, Cambridge : Polity Press, 1998, p. 11.

4. T. BLAIR, New Britain. My vision of a young country, Londres : Fourth Estate, 1996, pp. 16, 30-1.

5. NEC, Democratic Socialist Aims and Values, Londres : Labour Party, 1988.

6. T. BLAIR, New Britain, op.cit., p. 13.

7. Ibid., p. 4.

8. Ibid., p. 30.

9. P. MANDELSON \& L. MARTELL, The Blair Revolution, op.cit., p. viii.

10. Cité et traduit in Le Monde, 3 mai 1995, p. 6.

11. Tony Blair au Congrès de Blackpool, Le Monde, 6 octobre 1994.

12. T. BLAIR, New Britain, op.cit., p. 38.

13. Ibid., p. 7.

14. J. CROWLEY, Sans épines la rose. Tony Blair, un modèle pour l'Europe ?, Paris : La Découverte, 1999, pp. 19-20.

15. T. BLAIR, New Britain, op.cit., pp. 4-21.

16. LABOUR PARTY, Because Britain Deserves Better, Manifesto 1997, Londres : Labour Party, 1997, p. 5.

17. E. SHAW, The Labour Party since 1979, op.cit., p. 60.

18. Le Monde, 3 mai, 1995, p. 15 ; T. BLAIR, The Third Way. New Politics for the New Century, Londres : Fabian Society, 1998 ; M. CHRESTIEN (dir.), Le nouveau libéralisme anglais à l'aube du XXe siècle, Paris : Economica, 1999 ; A. GIDDENS, Beyond Left and Right. The future of radical politics, Cambridge : Polity Press, 1994 ; The Third Way. The renewal of social democracy, Cambridge : Polity Press, 1998 ; J. GRAY, Post Liberalism. Studies in political thought, Londres : Routledge, 1993 ; J. RAWLS, A Theory of Justice, Harvard : Harvard U.P., 1971 ; A. ETZIONI, The New Golden Rule: Community and Morality in a Democratic Society, New York: Basic Books, 1996; W. HUTTON, The State We're In, Londres: Vintage, 1996. Voir aussi les témoignages de T. Blair lui-même in New Britain, op.cit., pp. 57-61.

19. T. BLAIR, New Britain , op.cit., p. 289-321.

20. Ibid., p. xiii.

21. Le Monde, 6 mai 1997, p. 17 ; J. LERUEZ, Le Royaume Uni après les élections de mai 1997. Changement de gouvernement ou changement de régime?, Paris : Etudes du CERI, n³8, janvier 1998. 
22. A. SELDON (ed.), The Blair Effect. The Blair Government, 1997-2001, Londres : Little, Brown, 2001 ; J. RENTOUL, Tony Blair : Prime Minister, Londres : Little Brown, 2001. Pour un bilan plus politique voir LABOUR PARTY, Ambitions for Britain, Manifesto 2001, Londres : Labour Party, 2001, p. 44.

23. Les Echos, 25 mars 1998, p.6. Voir aussi P. MARLIERE, Le Blairisme, un thatchérisme à visage humain? in Les Temps Modernes, $\mathrm{n}^{\circ}$ 601, Paris, novembre 1998.

24. J. CROWLEY, Sans épines la rose, op.cit., p. 18.

25. Le Monde, 26-27 juillet 1998, p. 22.

26. Le Monde, 21 août 1998, p. 2.

27. Déclaration de P. MANDELSON.

28. Le Monde, 16 mai 1997.

29. A. GIDDENS in Le Monde, 6 octobre 1998, p. 1.

30. Congrès de Brighton. Le Monde, 2 octobre 1997.

31. Ibid.

32. P. MANDELSON, Notre révolution en marche, Le Monde, 21 septembre 2000. Sur les limites voir P. GOULD, The Unfinished Revolution. How the Modernisers Saved the Labour Party, Londres: Little Brown, 1998.

33. Voir la déclaration de Blair au Congrès de 1997: «Je veux que le XXI' siècle soit celui des radicaux». Voir également T. BLAIR, Le Royaume-Uni plus fort en Europe grâce à la Troisième Voie, Paris : Notes de la Fondation Jean Jaurès, n 13, août 1999.

34. Le Monde, 30 septembre 1999, p. 3.

35. Le Monde, 3 mai 1997, p. 15.

36. Le Monde, 2 octobre 1997.

37. The Guardian, 7 février 1998 ; Le Monde, 17 février 1998.

38. Le Monde, 23 septembre 1998, p. 4.

39. Le Monde, 30 septembre 1999, p. 3.

40. Le Monde, 26-27 juillet 1998, p. 22 ; 4 août 1998, p. 5 ; 20-21 juin 1999, p. 15 ; 30 novembre 1999 p. 10 ; 29 mai 2001, p. 2.

41. Le Monde, 10 juin 1999, p. 7 ; $1^{\mathrm{er}}$ octobre 1998, p. 2.

42. Le Monde, 14 juin et 31 octobre 2000.

43. Le Monde, 21-22 novembre 2001, p. 2.

44. Tout ceci en lien avec le nouveau think tank pour l'Europe : Policy Network. Le Monde, 23 février 200, p. 15.

45. J. DELORS in Le Monde, 3 mai 2000, p. III.

46. D. MACSHANE, L'euro doit changer l'Angleterre mais aussi l'Europe, Le Monde, 3 mars 1999, p. 14. Réponse de P. MARLIERE, Quelle Europe veut le New Labour?, Le Monde, 7-8 mars 1999, p. 12. Voir aussi A. MENON, Triomphant malgré lui, le Royaume-Uni et l'émergence de l'Europe anglo-saxonne, in Pouvoirs, $n^{\circ}$ 93, op.cit., pp. 177-193.

47. Le Monde, 6 juin 2001, p. 33.

48. The Economist, 9-16 juin 2001, pp. 47-49.

49. LABOUR PARTY, Ambitions for Britain, op.cit., pp. 3-5.

50. D. MARQUAND, "Le nouveau travaillisme ou les ambiguïtés de la modernité”, Pouvoirs, nº 93, op.cit., pp. 7-20. 


\section{RÉSUMÉS}

Le blairisme n'est pas vraiment une idéologie mais plutôt un code de valeurs qui emprunte sélectivement au travaillisme, au torysme et au radicalisme libéral pour les transcender en un syncrétisme modernisateur. Instrument tactique pour conquérir et garder le pouvoir, cette Troisième Voie s'autojustifie dans l'action, pour mieux rester fidèle à l'idéal du centrisme radical, tout en s'imposant comme le nouveau messianisme fédérateur de la construction européenne.

Blairism is not really an ideology, rather a value system borrowing selectively from Labourism, Torysm and radical Liberalism, transcending them in a modernizing gospel. A tactic to conquer and keep power, the Third Way legitimates itself through action in order to remain faithful to a "radicalism of the centre", as well as imposing itself as the new federative messianism of European construction.

\section{AUTEUR}

\section{FRANÇOIS-CHARLES MOUGEL}

Institut d'Études Politiques de Bordeaux 